\title{
Gland diseases: new perspectives in diagnostic radiology
}

Although a systematic approach remains crucial for an accurate diagnosis of gland disease, increased attention is paid mainly to the role of diagnostic radiology on its detection. In the last few years, the clinical benefits offered by different diagnostic tools have become essential for defining a still challenging condition which includes a broad spectrum of disease. Therefore, several inflammatory disorders, partly due to autoimmune diseases, could affect gland regions, other than anomalies of the cellularity, mainly due to tumors.

This special issue of "Gland Surgery" named "Diagnostic Radiology," quite clearly focuses on the latest update of radiologic approach to gland disease; however, it has not been an easy task.

Recent radiological scanners and technologies, including US technologies, have achieved a high diagnostic accuracy, which makes them an inextricable element of the clinical setting, both in routine clinical practice and in an emergency setting.

Robust current evidence also shows how radiological accuracy goes hand in hand with a strong prognostic validity, partly yet to be defined $(1,2)$.

Furthermore, the well-known interventional radiologist approach allows a minimally invasive treatment of different benign and malignant lesion (3).

Therefore, while radiology has become necessary, an all-encompassing understanding of the complex radiologic approaches remains quite difficult.

This issue is composed of 10 review articles and 2 original articles which is proposing to provide a comprehensive update and new evidence about the most important gland regions, including pituitary gland, thyroid, major salivary glands, thymus, breast, pancreas, adrenal gland, prostate, and testicles.

The reader can recognize these different trends in current diagnostic radiology: while some authors continue to stress the increasing accuracy of diagnostic tools in diagnosing still challenging disease, other authors successfully emphasized the new role of radiology in defining the correct risk stratification strategies and predictive validity of imaging.

The leading article belongs to the first cluster entitled "Diagnostic value of major salivary gland ultrasonography (SGUS) in primary Sjogren's syndrome: the role of grey-scale and color/power Doppler sonography" submitted by Salaffi et al. In this review, the Author offers a proper point of view about reliability and validity of SG-US. This is important evidence to discuss, considering the high correlation that SG-US has shown with other standard tests as minor salivary gland biopsy or unstimulated salivary flow $(4,5)$.

This review pairs well with the article submitted by Pradella et al. named "Groove Pancreatitis: a challenging imaging diagnosis", a not-widely-known and often misinterpreted condition which could mimic or can mask a consistent presence of pancreatic cancer $(6,7)$, which changes potential treatment and prognosis.

These interesting articles are not alone in accurately describing the radiological advantages in the study of gland diseases. For example, the paper titled, "High-resolution MR imaging at 3T of pituitary gland: advantages and pitfalls" submitted by Varrassi et al. describes advantages and pitfalls on pituitary gland study offered by the high magnetic field (8). Also, in "Update in diagnostic imaging of the thymus and anterior mediastinal masses" submitted by Gentili et al. it is well provided all necessary information for accurate differential diagnosis of mediastinal masses, considering the obvious clinical need in well identify the nature and aggressiveness of different conditions $(9,10)$.

Reginelli et al. analyze two other interesting arguments, through its "Delayed enhancement in the differential diagnosis of salivary gland neoplasm", which explains the multi-phasic CT usefulness in parotid gland lesions (11) as well as De Filippo et al. in "MR Imaging of adrenal gland: state of the art", which takes up the concept of highly advantageous tissue characterization offered by MRI, especially useful in a proper differential diagnosis of adrenal gland lesions (12).

The reader, otherwise, can approach other review articles using the second key of the lecture. Beyond the accuracy, it is necessary to recognize the prognostic validity of the radiologic information. Furthermore, the clinical utility derives from both diagnostic accuracy and prognostic validity. Therefore, the use of the proper diagnostic tools could be mandatory for an appropriate therapeutic approach.

In this effort, another article titled "Neuroblastoma image-defined risk factors in adrenal neuroblastoma: the role of radiologist" submitted by Lanza et al., emphasizes the role of the radiologist in providing necessary information for the proper 
preoperative staging of adrenal neuroblastoma (13-15). Alternatively, in "MRI predictive role in the therapeutic response of GH-secreting pituitary adenomas" submitted by Caranci et al., clearly describes that MRI information is necessary for the best treatment (e.g., previous described potential fibrous characteristic, its extension in the cavernous sinus, etc.) $(16,17)$.

Similarly, the two other articles focus on topic of particular interest in the current radiologic scenario; on the one hand, "Correlation between ADC values and Gleason score in evaluation of prostate cancer: multicenter experience and review of the literature" submitted by Manetta et al., which approaches to tumor grading, the most important factor in the predictive analysis $(18,19)$.

On the other hand, "Ultrasound imaging classification of thyroid nodules for malignancy risk stratification and clinical management: state of the art" submitted by Floridi et al., which enhances the role of the radiologist in defining a characteristic of nodule malignancy $(20,21)$. Thyroid nodules are very common and an appropriate risk definition is mandatory (22).

At the end, two other articles deserve a particular mention: "Does multiparametric US improve diagnostic accuracy in the characterization of small testicular masses" submitted by Reginelli et al., and "Hereditary breast cancer: screening and risk-reducing surgery" submitted by Clemente et al.; this original article could improve current knowledge on gland disease, particularly through the application of an elastography in the scrotal US (23) and through the use of MRI in high-risk breastcancer patients for the detection of very small breast cancer (24).

To conclude, we hope that this discussed topic could be useful to radiologists as well as to another clinicians, specialists or whatnot, in defining an accurate diagnostic and management algorithm of gland disease.

We appreciate the intensive work of all authors, who have tried to provide a proper review of the latest imaging update and news on gland disease, to discuss the current "check-point."

\section{Acknowledgments}

None.

\section{References}

1. Cortellini A, Palumbo P, Porzio G, et al. Single-institution study of correlations between skeletal muscle mass, its density, and clinical outcomes in non-small cell lung cancer patients treated with first-line chemotherapy. Thorac Cancer 2018;9:1623-30.

2. Lai Q, Nicolini D, Inostroza Nunez M, et al. A Novel Prognostic Index in Patients With Hepatocellular Cancer Waiting for Liver Transplantation. Ann Surg 2016;264:787-96.

3. Barile A, Arrigoni F, Bruno F, et al. Present role and future perspectives of interventional radiology in the treatment of painful bone lesions. Future Oncol 2018;14:2945-55.

4. Baldini C, Luciano N, Tarantini G, et al. Salivary gland ultrasonography: A highly specific tool for the early diagnosis of primary Sjögren's syndrome. Arthritis Res Ther 2015;17:146-8.

5. Jousse-Joulin S, Nowak E, Cornec D, et al. Salivary gland ultrasound abnormalities in primary Sjögren's syndrome: Consensual US-SG core items definition and reliability. RMD Open 2017;3:e000364.

6. Pallisera-Lloveras A, Ramia-Ángel JM, Vicens-Arbona C, et al. Groove pancreatitis. Rev Esp Enferm Dig 2015;107:280-8.

7. Raman SP, Salaria SN, Hruban RH, et al. Groove pancreatitis: Spectrum of imaging findings and radiology-pathology correlation. AJR Am J Roentgenol 2013;201:W29-39.

8. Nakazawa H, Hashizume C, Shibamoto $\mathrm{Y}$, et al. Efficacy of magnetic resonance imaging at $3 \mathrm{~T}$ compared with $1.5 \mathrm{~T}$ in small pituitary tumors for stereotactic radiosurgery planning. Jpn J Radiol 2014;32:22-9.

9. Yabuuchi H, Matsuo Y, Abe K, et al. Anterior mediastinal solid tumours in adults: Characterisation using dynamic contrastenhanced MRI, diffusion-weighted MRI, and FDG-PET/CT. Clin Radiol 2015;70:1289-98.

10. Ackman JB, Verzosa S, Kovach AE, et al. High rate of unnecessary thymectomy and its cause. Can computed tomography distinguish thymoma, lymphoma, thymic hyperplasia, and thymic cysts? Eur J Radiol 2015;84:524-33.

11. Metwally Abo El Atta M, Ahmed Amer T, Mohamed Gaballa G, et al. Multi-phasic CT versus dynamic contrast enhanced MRI in characterization of parotid gland tumors. Egypt J Radiol Nucl Med 2016;47:1361-72.

12. Elsayes KM, Mukundan G, Narra VR, et al. Adrenal Masses: MR Imaging Features with Pathologic Correlation. Radiographics 
2004;24:S73-86.

13. Chen AM, Trout AT, Towbin AJ. A review of neuroblastoma image-defined risk factors on magnetic resonance imaging. Pediatr Radiol 2018;48:1337-47.

14. Brisse HJ, McCarville MB, Granata C, et al. Guidelines for Imaging and Staging of Neuroblastic Tumors: Consensus Report from the International Neuroblastoma Risk Group Project. Radiology 2011;261:243-57.

15. McCarville MB. Imaging neuroblastoma: What the radiologist needs to know. Cancer Imaging 2011;11 Spec No A:S44-7.

16. Boxerman JL, Rogg JM, Donahue JE, et al. Preoperative MRI Evaluation of Pituitary Macroadenoma: Imaging Features Predictive of Successful Transsphenoidal Surgery. AJR Am J Roentgenol 2010;195:720-8.

17. Wang F, Zhang J, Wang P, et al. Prediction of pituitary stalk position in pituitary adenomas by visualization of the hypothalamohypophyseal tract using diffusion tensor imaging tractography. Medicine (Baltimore) 2018;97:e0052.

18. Alessandrino F, Taghipour M, Hassanzadeh E, et al. Predictive role of PI-RADSv2 and ADC parameters in differentiating Gleason pattern $3+4$ and $4+3$ prostate cancer. Abdom Radiol (NY) 2019;44:279-85.

19. Boesen L, Chabanova E, Løgager V, et al. Apparent diffusion coefficient ratio correlates significantly with prostate cancer gleason score at final pathology. J Magn Reson Imaging 2015;42:446-53.

20. Russ G, Durante C, Leenhardt L, et al. European Thyroid Association Guidelines for Ultrasound Malignancy Risk Stratification of Thyroid Nodules in Adults: The EU-TIRADS. Eur Thyroid J 2017;6:225-37.

21. Smith-Bindman R, Lebda P, Feldstein VA, et al. Risk of Thyroid Cancer Based on Thyroid Ultrasound Imaging Characteristics. JAMA Intern Med 2013;173:1788.

22. Brunese L, Romeo A, Iorio S, et al. Thyroid B-flow twinkling sign: a new feature of papillary cancer. Eur J Endocrinol 2008;159:447-51.

23. Aigner F, De Zordo T, Pallwein-Prettner L, et al. Real-time sonoelastography for the evaluation of testicular lesions. Radiology 2012;263:584-9.

24. Lee MV, Katabathina VS, Bowerson ML, et al. BRCA -associated Cancers: Role of Imaging in Screening, Diagnosis, and Management. RadioGraphics 2017;37:1005-23.

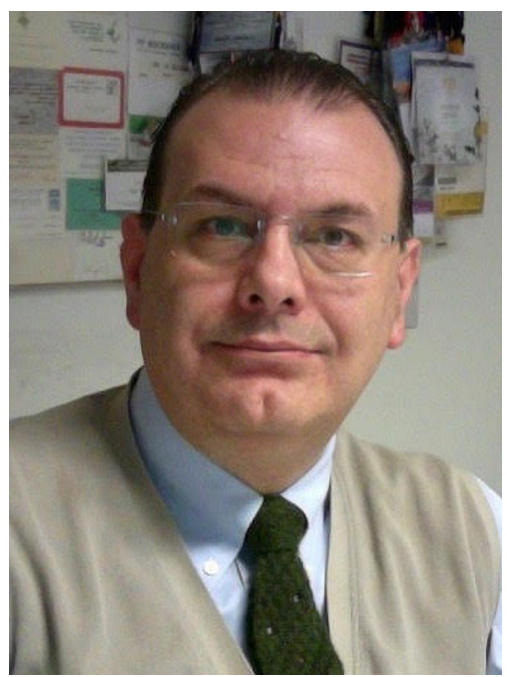

Antonio Barile

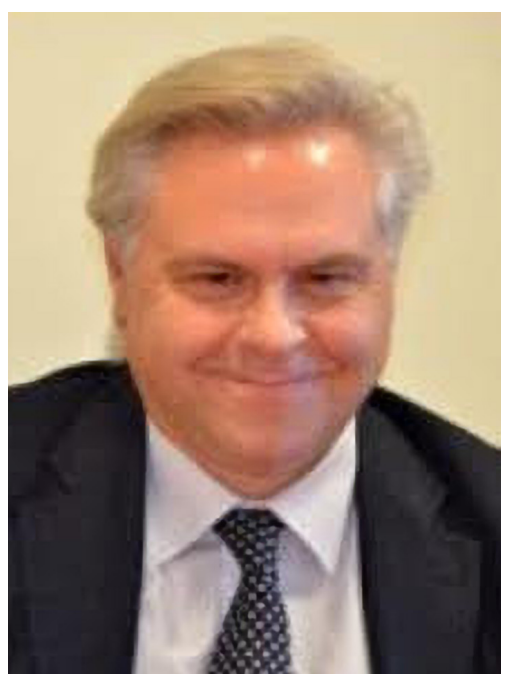

Luca Brunese

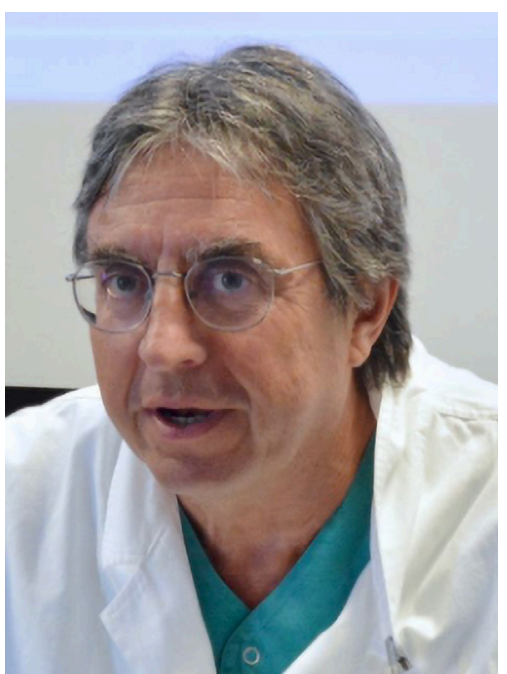

Andrea Giovagnoni 
Antonio Barile, MD

Department of Biotechnology and Applied Clinical Sciences, University of L'Aquila, L'Aquila, Italy.

(Email: antonio.barile@cc.univaq.it)

Luca Brunese, MD

Department of Medicine and Health Sciences "V. Tiberio", University of Molise, Campobasso, Italy.

(Email: luca.brunese@unimol.it)

Andrea Giovagnoni, MD

Department of Radiology, Ospedali Riuniti, Università Politecnica Delle Marche, Ancona, Italy.

(Email:a.giovagnoni@univpm.it) doi: $10.21037 / \mathrm{gs} .2019 .03 .05$

Conflicts of Interest: The authors have no conflicts of interest to declare.

View this article at: http://dx.doi.org/10.21037/gs.2019.03.05

Cite this article as: Barile A, Brunese L, Giovagnoni A. Gland diseases: new perspectives in diagnostic radiology. Gland Surg 2019;8(Suppl 3):S126-S129. doi: 10.21037/gs.2019.03.05 